Boogaard, E. van den, Cohn, D.M., Korevaar, J.C., Dawood, F., Vissenberg, R., Middeldorp, S., Goddijn, M., Farquharson, R.G. Number and sequence of preceding miscarriages and maternal age for the prediction of antiphospholipid syndrome in women with recurrent miscarriage 7 Fertility and Sterility: 2013, 99(1), 188-192

\begin{tabular}{|l|l|}
$\begin{array}{l}\text { Postprint } \\
\text { Version }\end{array}$ & 1.0 \\
\hline Journal website & http://www.sciencedirect.com/science/article/pii/S0015028212021802 \\
\hline Pubmed link & $\underline{\text { http://www.ncbi.nlm.nih.gov/pubmed/23043688 }}$ \\
\hline DOI & $10.1016 /$ j.fertnstert.2012.09.002 \\
\hline
\end{tabular}

This is a NIVEL certified Post Print, more info at http://www.nivel.eu

\title{
Number and sequence of preceding miscarriages and maternal age for the prediction of antiphospholipid syndrome in women with recurrent miscarriage
}

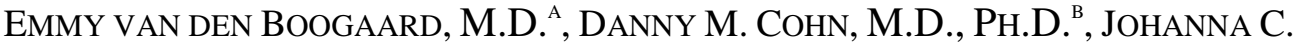 \\ Korevaar, Ph.D. ', Feroza Dawood, M.D., Ph.D. ${ }^{\mathrm{D}}$, Rosa Vissenberg, M.D. ${ }^{\mathrm{A}}$, Saskia \\ Middeldorp, M.D., Ph.D. ${ }^{\mathrm{B}}$, Mariette Goddijn, Ph.D. ${ }^{\mathrm{A}}$, Roy G. Farquharson, M.D. ${ }^{\mathrm{D}}$ \\ ${ }^{a}$ Center for Reproductive Medicine, Academic Medical Center, Amsterdam, the Netherlands \\ ${ }^{b}$ Department of Vascular Medicine, Academic Medical Center, Amsterdam, the Netherlands \\ ${ }^{\mathrm{c}}$ Department of Clinical Epidemiology, Academic Medical Center, Amsterdam, the \\ Netherlands \\ ${ }^{d}$ Department of Obstetrics and Gynaecology, Liverpool Women's Hospital, Liverpool, United \\ Kingdom
}

Objective

To investigate the relationship between the number and sequence of preceding miscarriages and antiphospholipid syndrome (APS).

Design

Retrospective cohort study.

Setting

Recurrent miscarriage (RM) clinic.

Patient(s)

Women who attended the RM clinic from 1988 to 2006.

Intervention(s)

None.

Main Outcome Measure(s)

Number, type, and sequence of previous pregnancies were compared between women with APS and women with unexplained RM.

Result(s)

A total of 1,719 patients were included; 312 (18\%) had APS, and 1,407 (82\%) had unexplained RM. The mean maternal age (32.6 years) did not differ between women with and without APS. The median number of miscarriages was three in both groups. A total of 865 women (50\%) had a history of at least one live birth, with no difference between the two groups. In both groups, $97 \%$ of the women had a history of consecutive miscarriages. 
Boogaard, E. van den, Cohn, D.M., Korevaar, J.C., Dawood, F., Vissenberg, R., Middeldorp, S." Goddijn, M., Farquharson, R.G. Number and sequence of preceding miscarriages and maternal age for the prediction of antiphospholipid syndrome in women with recurrent miscarriage 7 Fertility and Sterility: 2013, 99(1), 188-192

Conclusion(s)

The number of preceding miscarriage, type and sequence of previous pregnancies, and maternal age were not associated with APS in women with RM. There is no increased diagnostic yield for APS after three miscarriages rather than after two miscarriages and no increased diagnostic yield for APS after consecutive miscarriages rather than after nonconsecutive miscarriages. Therefore, APS testing should be considered for all women with two or more miscarriages.

Of all couples trying to conceive, $2 \%-4 \%$ are confronted with recurrent miscarriage (RM) 1 and 2. RM is characterized by a heterogeneous population, and in more than $50 \%$ of the couples with RM the cause of their miscarriages remains unexplained 2, 3 and 4. Risk factors for RM include maternal age, parental structural chromosomal abnormality, anatomic and hormonal abnormalities, and thrombophilia 5, 6, 7 and 8. It is known that too many diagnostic tests are performed in couples with RM, and there is a lively debate whether diagnostic tests should be performed in all women with RM or if a more efficient approach to test only in subgroups at high risk for an underlying abnormality should be applied (7).

Antiphospholipid syndrome (APS) is an acquired thrombophilia and an established cause of RM 9, 10, 11 and 12. It is internationally defined as the presence of thrombosis, pregnancy loss, or maternal morbidity and persistent circulating antiphospholipid antibodies (APLAs) in plasma 13 and 14. The incidence of APS in women with RM is reported to be $\sim 15 \%$ (15). The overall prognosis of a successful pregnancy in women with RM and APLAs is good, and is possibly improved when treated with heparin and aspirin 10,16 and 17. It is recommended that testing for lupus anticoagulants and anticardiolipin antibodies should occur in women with a history of two to three or more early pregnancy losses before 10 weeks, or one or more unexplained deaths at $\geq 10$ weeks of a morphologically normal fetus $\underline{8}, 18,19$ and 20. RM is internationally defined as two to three or more miscarriages, sometimes defined to be consecutive.

Many articles focus on therapeutic options for patients with APS and RM, and relatively little effort has been put into improving the diagnostic accuracy in APS and RM (21). Jaslow et al. (22) showed that women with two consecutive miscarriages are at the same risk for APS as women with three or more consecutive miscarriages. At present, it is unknown whether the diagnostic yield of testing for APS can be increased by clinical determinants derived from the extensive obstetric history. Current debates about starting diagnostics after two or three miscarriages or taking into account maternal age have not been resolved for APS.

The aim of the present study was to investigate the relationship between characteristics of preceding miscarriages and maternal age in couples with RM and a diagnosis of APS to define a risk profile to determine the efficacy of testing for APS.

\section{MATERIALS AND METHODS}

A cohort study was performed including all women with two or more miscarriages who attended the RM clinic of Liverpool Women's Hospital, Liverpool, U.K., from 1988 to 2006. All women were prospectively included in the database from 1988. The present study was defined retrospectively, and analyses were performed retrospectively. Miscarriage was defined as a pregnancy loss at $<24$ weeks of 
Boogaard, E. van den, Cohn, D.M., Korevaar, J.C., Dawood, F., Vissenberg, R., Middeldorp, S." Goddijn, M., Farquharson, R.G. Number and sequence of preceding miscarriages and maternal age for the prediction of antiphospholipid syndrome in women with recurrent miscarriage 7 Fertility and Sterility: 2013, 99(1), 188-192

gestation. Miscarriage was preferably clinically proven by an early ultrasound, but at least with a positive pregnancy test (National Guideline Clearinghouse). All data entries were verified by a second independent local investigator to ensure accuracy of data entry in the database. Data were collected prospectively for research purposes. Our research question was developed afterwards, so the design is considered to be a retrospective cohort study. An extra random data validity check, i.e., a random selection of medical files, was checked for consistency with the database when the present study was performed in 2010 by two external researchers (E.v.d.B. and D.C.). Data on preceding pregnancies were collected: number of previous pregnancies, type of pregnancy, and the sequence. Miscarriages were considered to be consecutive when not interspersed with a successful pregnancy. All patients underwent a systematic diagnostic work-up including testing for APS, screening for thrombophilia (APLAs and acquired activated protein $C$ resistance), chromosome abnormalities (in both partners), thyroid dysfunction, diabetes mellitus, bacterial vaginosis, and uterine abnormalities. Exclusion criteria were established causes for RM other than APS: a parental chromosomal abnormality, major (congenital) uterine abnormalities, endocrine disorders at the time of previous miscarriages (including diabetes mellitus and thyroid dysfunction), pregnancy losses due to documented fetal malformation or autoimmune disorders (e.g., systemic lupus erythematosus and thrombophilia). Patients with an incomplete dataset or missing test results were also excluded.

The diagnostic criteria for APLAs have been applied in previously reported studies from the same center 9, 17, 23, 24, 25 and 26. Lupus anticoagulant was confirmed by the dilute Russell's viper venom time (DRVVT) with a neutralization procedure using frozen thawed platelets. The upper limit of the normal range for anticardiolipin antibodies and DRVVT was determined from the log-transformed mean plus 2 standard deviations of results in 50 healthy nonpregnant adults. Women were qualified as anticardiolipin antibody positive if they met the following criteria: IgM anticardiolipin antibodies $\geq 6 \mathrm{U} / \mathrm{mL}$, IgG anticardiolipin antibodies $\geq 11 \mathrm{U} / \mathrm{mL}$, and/or DRVVT $\geq 1.10$ with a neutralization procedure using frozen-thawed platelets. All of the samples were analyzed in the same laboratory according to a standardized protocol. APLAs were considered to be present if a test was positive on two occasions $\geq 6$ weeks apart. Those women were diagnosed as APS positive. Characteristics of women with proven APS according to the Sapporo criteria were compared with women with unexplained RM $\underline{13}$ and $\underline{14}$. The main outcome measure was the presence of APLAs. Independent variables were maternal age at the time of presentation, number of preceding miscarriages, first-trimester and second-trimester miscarriages, the presence and number of preceding live births at $>24$ weeks gestational age, and the sequence (consecutive vs. nonconsecutive) of miscarriages. The study was approved by the Institutional Review Board of the Liverpool Women's Hospital.

\section{STATISTICAL ANALYSIS}

Univariable and multivariable binary logistic regression analyses were performed to calculate differences between the two groups, women with RM and APS and women with unexplained RM. Subgroup analyses were performed on patients with at least three preceding consecutive and nonconsecutive miscarriages. We used SPSS software (version 16.0) to perform the analyses. 
Boogaard, E. van den, Cohn, D.M., Korevaar, J.C., Dawood, F., Vissenberg, R., Middeldorp, S." Goddijn, M., Farquharson, R.G. Number and sequence of preceding miscarriages and maternal age for the prediction of antiphospholipid syndrome in women with recurrent miscarriage 7 Fertility and Sterility: 2013, 99(1), 188-192

\section{RESULTS}

During the study period, 2,055 couples were analyzed for RM. After applying the exclusion criteria, 336 were excluded from further analyses: 69 patients did not meet the criteria for RM, and in 267 patients one or more other causes for their RM were found. Among the excluded patients were 78 patients with APS combined with another risk factor established for RM. Those patients were excluded to avoid confounders in our analysis. Inherited thrombophilia was diagnosed in 30 patients. Of those, three patients (10\%) were also positive for antiphospholipid antibodies. The presence of inherited thrombophilia proved to not to be associated with APS compared with unexplained RM (odds ratio [OR] 0.87, 95\% confidence interval [CI] 0.87-10).

Data from 1,719 patients fulfilled the inclusion criteria for analysis (Fig. 1). Of those patients, 312 (18\%) had APS and 1,407 (82\%) had unexplained RM. The mean maternal age at the time of presentation was 32.6 years (SD 5.8). The median number of preceding miscarriages was three (range 2-14); 527 patients (31\%) had a history of two miscarriages, and 1,192 (69\%) had experienced three or more miscarriages. Of the included patients, 1,363 (79\%) experienced only first-trimester miscarriages, 51 (3\%) only second-trimester miscarriages, and 305 (18\%) both. Before their first visit, one-half of the patients, 865 (50\%), had at least one live birth after 24 weeks of gestation. The mean number of preceding live births was 0.71 (SD 0.91).

\section{[FIGURE 1]}

Differences between patients with APLAs and patients with unexplained RM are presented in Table 1. Maternal age at the time of presentation (OR 1.00, 95\% CI 0.98-1.02) and the obstetric history did not differ between the two groups. The number of preceding miscarriages and live births was equally distributed among patients with APS and with unexplained RM (OR 1.02, 95\% CI 0.94-1.10; and OR 1.06, 95\% CI 0.92-1.21; respectively).

\section{[TABLE 1]}

The sequence of preceding pregnancies was known in 1,526 patients (89\%): 265 APS-positive patients and 1,261 patients with unexplained RM. The majority of patients had experienced consecutive miscarriages (97\% and 98\%, respectively). This was not different between patients with and without APS (OR 0.73, 95\% CI 0.33-1.62; Table 1). In those patients with a history of at least three miscarriages (n $=1,192)$, the sequence of preceding pregnancies was recorded in $918(77 \%)$. The prevalence of consecutive miscarriages was similar in patients with three miscarriages and APS to that in patients with three or more unexplained miscarriages (OR 0.84, 95\% CI 0.50-1.44; Table 2). The prevalence of APS in the group of women to 2 or more miscarriages was $17.4 \%(265 / 1,526)$. The prevalence of APS in the group of women with three or more miscarriages was $17.3 \%(159 / 918)$. The incidence of APS did not differ among ages. Multivariable logistic regression analyses, including all independent variable on maternal and obstetric history, showed no significant differences between women with and without APS for any of the variables.

\section{[TABLE 2]}


Boogaard, E. van den, Cohn, D.M., Korevaar, J.C., Dawood, F., Vissenberg, R., Middeldorp, S." Goddijn, M., Farquharson, R.G. Number and sequence of preceding miscarriages and maternal age for the prediction of antiphospholipid syndrome in women with recurrent miscarriage

Fertility and Sterility: 2013, 99(1), 188-192

\section{DISCUSSION}

In this large cohort study we showed that patients with RM and APS did not differ for clinical obstetric parameters compared with patients with unexplained RM. Maternal age at the time of presentation and number, type, and sequence of preceding pregnancies were not found to be independent variables for the presence of APLAs in patients with RM.

Identification of patients with APS is important, because several studies have suggested the benefit of anticoagulant treatment in patients with RM and APS. Future live birth rates can possibly be increased from $40 \%$ to $70 \%$ when treatment during the next pregnancy is initiated 9, $\underline{11}, \underline{12}, \underline{17}, \underline{18}, 27,28$ and 29. Because the results of published trials so far are conflicting, further research on the therapeutic pathway for patients with RM and APS is still urgently needed.

When studying RM and APS, the first hurdle is the correct definition of these clinical conditions. International definitions of recurrent miscarriage vary in their number (two vs. three or more) of miscarriages and in the outcome and sequence (consecutive vs. nonconsecutive) of preceding pregnancies $\underline{8}, \underline{19}, 30$ and 31 . Regarding the number of preceding miscarriages necessary to meet the criteria of $\mathrm{RM}$, this has previously been addressed in studies regarding carrier status of chromosomal abnormalities in these couples (7). The number of preceding miscarriages proved to be an independent risk factor for a parental chromosome abnormality: The chance of a parental chromosome abnormality was higher after three miscarriages compared with two miscarriages. Our results indicate that the number of miscarriages (two vs. three) is not an independent risk factor for APS. One-third of the included patients had a history of two miscarriages, and their risk for APS was similar to those patients with a history of three or more miscarriages. Jaslow et al. (22) also confirmed that the incidence of abnormal results for evidencebased and investigative diagnostic tests did not differ among women with different numbers of pregnancy losses. Our results for APS are in line with that study: Couples with two miscarriages as well as three or more miscarriages have an increased risk for underlying pathology. Another study also reported that prior pregnancy loss and maternal age did not predict for APS (32).

The sequence of preceding pregnancies, i.e., whether the miscarriages were consecutive or nonconsecutive, is a more uncultivated field of research. This aspect of the definition of RM in guidelines seems to be more instinctively driven than evidence based. This topic has previously been studied in relation to chromosomal abnormalities, where no effect of sequence could be found (20). In our patients, the sequence of preceding pregnancies was also not associated with the risk of having APS. We would therefore recommend offering testing for APLAs to patients with nonconsecutive miscarriages, to allow further examination of the relevance of sequence in underlying pathology. The type of pregnancy loss history, e.g., loss of fetal heart activity, is often described to be associated with APS. Older publications showed prolonged pregnancy duration and later loss of fetal heart activity in APS compared with non-APS patients (33). Although the presence or absence of a fetal heart beat was not documented in our database, there was no difference in first- or second-trimester losses between APS and non-APS patients.

The strength of the present study is the large number of women with RM and APS, which provided us with the ability to investigate many aspects of the obstetric history and to perform subgroup analyses on women with two or three or more miscarriages. 
Boogaard, E. van den, Cohn, D.M., Korevaar, J.C., Dawood, F., Vissenberg, R., Middeldorp, S." Goddijn, M., Farquharson, R.G. Number and sequence of preceding miscarriages and maternal age for the prediction of antiphospholipid syndrome in women with recurrent miscarriage 7 Fertility and Sterility: 2013, 99(1), 188-192

The latter is important because of the ongoing debate on the international definition of RM. The evidence from this cohort study (level IIb) can be included in criteria which have been consensus based (14). With these data at hand, the criteria for APS screening could be changed into "two or more (not necessarily consecutive) firsttrimester miscarriages.” All measurements were performed in the same laboratory; thus it is a homogeneous cohort despite the long time span of data collection. Some limitations should be considered when interpreting the results of this study. First, the cut-off point for positive anticardiolipin antibodies is lower than stated in the most updated criteria 14 and 34. Patients from this cohort were screened according to the Sapporo criteria (13). Nevertheless, therapeutic success, i.e., higher live birth rates, has been reported in even lower cut-off values (2). Further trials should define subgroup analyses on patients with high antibody levels to be able to refine the definition of APS in RM. The more recent anti- $\beta 2$-glycoprotein antibodies could not be included, because no measurement was performed in our patients. These antibodies are not yet incorporated in RM diagnostic work-up, because their relationship to RM is so far not clear but might be an additional important factor in future research on this topic. The long study period makes the set of data prone to incompleteness, which limits the analysis owing to missing data, but we were still able to collect a large cohort. Data were collected prospectively, all data entries were double-checked, and a random validity check was performed before analysis. We therefore considered the quality of the data to be ensured and the results widely applicable.

An individualized risk estimation for APS in couples with RM and other preceding adverse pregnancy outcomes, such as prematurity, small for gestational age, preeclampsia, and placental insufficiency, could be of additional value (32). These data were, however, not available in the database.

In conclusion, neither the number of miscarriages and the sequence of pregnancies nor maternal age differed with the presence of APS in couples with RM. Therefore, these clinical parameters prove that it is justifiable to offer testing for APS to all patients with a history of two or more, consecutive or nonconsecutive, miscarriages.

\section{ACKNOWLEDGMENTS}

The authors acknowledge Linda Roberts, without whom the data collection never would have been successfully completed. Furthermore, they acknowledge the Information Technology department of Liverpool Women's Hospital for their assistance.

\section{REFERENCES}

1L. Regan, R. RaiEpidemiology and the medical causes of miscarriageBaillieres Best Pract Res Clin Obstet Gynaecol, 14 (2000), pp. 839-854

2R. Rai, L. ReganRecurrent miscarriageLancet, 368 (2006), pp. 601-611

3G.M. StirratRecurrent miscarriage. II: Clinical associations, causes, and managementLancet, 336 (1990), pp. 728-733

| 4M.D. StephensonFrequency of factors associated with habitual abortion in 197 couplesFertil Steril, 66 (1996), pp. 24-

5A.M. Nybo Andersen, J. Wohlfahrt, P. Christens, J. Olsen, M. MelbyeMaternal age and fetal loss: population based register linkage studyBMJ, 320 (2000), pp. 1708-1712 
Boogaard, E. van den, Cohn, D.M., Korevaar, J.C., Dawood, F., Vissenberg, R., Middeldorp, S." Goddijn, M., Farquharson, R.G. Number and sequence of preceding miscarriages and maternal age for the prediction of antiphospholipid syndrome in women with recurrent miscarriage 7 Fertility and Sterility: 2013, 99(1), 188-192

6O.B. Christiansen, A.M. Nybo Andersen, E. Bosch, S. Daya, P.J. Delves, T.V. Hviid et nivel

al.Evidence-based investigations and treatments of recurrent pregnancy lossFertil Steril, 83 (2005), pp. 821-839

7M.T. Franssen, J.C. Korevaar, F. van der Veen, K. Boer, N.J. Leschot, M. GoddijnManagement of recurrent miscarriage: evaluating the impact of a guidelineHum Reprod, 22 (2007), pp. 1298-1303

8E. Jauniaux, R.G. Farquharson, O.B. Christiansen, N. ExaltoEvidence-based guidelines for the investigation and medical treatment of recurrent miscarriageHum Reprod, 21 (2006), pp. 2216-2222

9R.G. Farquharson, S. Quenby, M. GreavesAntiphospholipid syndrome in pregnancy: a randomized, controlled trial of treatmentObstet Gynecol, 100 (2002), pp. 408-413

10M. Empson, M. Lassere, J. Craig, J. ScottPrevention of recurrent miscarriage for women with antiphospholipid antibody or lupus anticoagulantCochrane Database Syst Rev (2005), p. CD002859

11W.H. Kutteh, L.D. ErmelA clinical trial for the treatment of antiphospholipid antibodyassociated recurrent pregnancy loss with lower dose heparin and aspirinAm J Reprod Immunol, 35 (1996), pp. 402-407

12L.S. Noble, W.H. Kutteh, N. Lashey, R.D. Franklin, J. HerradaAntiphospholipid antibodies associated with recurrent pregnancy loss: prospective, multicenter, controlled pilot study comparing treatment with low-molecular-weight heparin versus unfractionated heparinFertil Steril, 83 (2005), pp. 684-690

13W.A. Wilson, A.E. Gharavi, T. Koike, M.D. Lockshin, D.W. Branch, J.C. Piette et al.International consensus statement on preliminary classification criteria for definite antiphospholipid syndrome: report of an international workshopArthritis Rheum, 42 (1999), pp. 1309-1311

14S. Miyakis, M.D. Lockshin, T. Atsumi, D.W. Branch, R.L. Brey, R. Cervera et al.International consensus statement on an update of the classification criteria for definite antiphospholipid syndrome (APS)J Thromb Haemost, 4 (2006), pp. 295-306

15R.S. Rai, L. Regan, K. Clifford, W. Pickering, M. Dave, I. Mackie et al.Antiphospholipid antibodies and beta 2-glycoprotein-I in 500 women with recurrent miscarriage: results of a comprehensive screening approachHum Reprod, 10 (1995), pp. 2001-2005

16C.A. Laskin, C. Bombardier, M.E. Hannah, F.P. Mandel, J.W. Ritchie, V. Farewell et al.Prednisone and aspirin in women with autoantibodies and unexplained recurrent fetal lossN Engl J Med, 337 (1997), pp. 148-153

17D.M. Cohn, M. Goddijn, S. Middeldorp, J.C. Korevaar, F. Dawood, R.G. FarquharsonRecurrent miscarriage and antiphospholipid antibodies: prognosis of subsequent pregnancyJ Thromb Haemost, 8 (2010), pp. 2208-2213

18R. Rai, H. Cohen, M. Dave, L. ReganRandomised controlled trial of aspirin and aspirin plus heparin in pregnant women with recurrent miscarriage associated with phospholipid antibodies (or antiphospholipid antibodies)BMJ, 314 (1997), pp. 253-257

19National Guideline Clearinghouse (NGC). Guideline recurrent miscarriage. November 2009. Available at: http://www.guideline.gov/content.aspx?id=145712012. Translated from the Dutch Guideline on Recurrent Miscarriage.

20E. van den Boogaard, M. Goddijn, N.J. Leschot, F. van der Veen, J.A. Kremer, R.P. HermensDevelopment of guideline-based quality indicators for recurrent miscarriageReprod Biomed Online, 20 (2010), pp. 267-273

21D.W. Branch, M. Gibson, R.M. SilverClinical practice. Recurrent miscarriageN Engl J Med, 363 (2010), pp. 1740-1747

22C.R. Jaslow, J.L. Carney, W.H. KuttehDiagnostic factors identified in 1020 women with two versus three or more recurrent pregnancy lossesFertil Steril, 193 (2010), pp. 12341243

23A.J. Drakeley, S. Quenby, R.G. FarquharsonMid-trimester loss—appraisal of a screening protocolHum Reprod, 13 (1998), pp. 1975-1980

24S. Quenby, R.G. Farquharson, F. Dawood, A.M. Hughes, J. ToppingRecurrent miscarriage and long-term thrombosis risk: a case-control studyHum Reprod, 20 (2005), pp. 1729-1732 
Boogaard, E. van den, Cohn, D.M., Korevaar, J.C., Dawood, F., Vissenberg, R., Middeldorp, S." Goddijn, M., Farquharson, R.G. Number and sequence of preceding miscarriages and maternal age for the prediction of antiphospholipid syndrome in women with recurrent miscarriage 7 Fertility and Sterility: 2013, 99(1), 188-192

25J. Topping, S. Quenby, R. Farquharson, R. Malia, M. GreavesMarked variation in antiphospholipid antibodies during pregnancy: relationships to pregnancy outcomeHum Reprod, 14 (1999), pp. 224-228

26S.A. Brigham, C. Conlon, R.G. FarquharsonA longitudinal study of pregnancy outcome following idiopathic recurrent miscarriageHum Reprod, 14 (1999), pp. 2868-2871

27C.A. Laskin, K.A. Spitzer, C.A. Clark, M.R. Crowther, J.S. Ginsberg, G.A. Hawker et al. Low molecular weight heparin and aspirin for recurrent pregnancy loss: results from the randomized, controlled HepASA trialJ Rheumatol, 36 (2009), pp. 279-287

28W.H. KuttehAntiphospholipid antibody-associated recurrent pregnancy loss: treatment with heparin and low-dose aspirin is superior to low-dose aspirin aloneAm J Obstet Gynecol, 174 (1996), pp. 1584-1589

29S. Cowchock, E.A. Reece, Organizing Group of the Antiphospholipid Antibody Treatment TrialDo low-risk pregnant women with antiphospholipid antibodies need to be treated?Am J Obstet Gynecol, 176 (1997), pp. 1099-1100

30Royal College of Obstetricians and Gynaecologists. The investigation and treatment of couples with recurrent miscarriage. (Green-top 17.) May 2011. Available at: http://www.rcog.org.uk. Last accessed October 8, 2012.

31Definitions of infertility and recurrent pregnancy lossFertil Steril, 90 (2008), p. S60

32M.D. Lockshin, M. Kim, C.A. Laskin, M. Guerra, D.W. Branch, J. Merrill et al.Lupus anticoagulant, but not anticardiolipin antibody, predicts adverse pregnancy outcome in patients with antiphospholipid antibodiesArthritis Rheum, 64 (2012), pp. 2311-2318

33L.W. Chamley, A.M. Duncalf, M.D. Mitchell, P.M. JohnsonAction of anticardiolipin and antibodies to beta2-glycoprotein-I on trophoblast proliferation as a mechanism for fetal deathLancet, 352 (1998), pp. 1037-1038

34M. Galli, G. Reber, P. de Moerloose, P.G. de GrootInvitation to a debate on the serological criteria that define the antiphospholipid syndromeJ Thromb Haemost, 6 (2008), pp. 399401

\section{TABLES AND FIGURES}

\section{[TABLE 1]}

Characteristics of obstetric history in women with antiphospholipid syndrome (APS) and unexplained recurrent miscarriage (RM).

\begin{tabular}{|l||l||l|l|}
\hline \hline Independent variable & $\begin{array}{l}\text { Women with } \\
\text { APS (n= 301) }\end{array}$ & $\begin{array}{l}\text { Women with } \\
\text { unexplained RM (n }= \\
\mathbf{1 , 4 0 7})\end{array}$ & $\begin{array}{l}\text { Odds ratio } \\
\mathbf{9 5 \%} \mathbf{C I})^{\mathbf{a}}\end{array}$ \\
\hline $\begin{array}{l}\text { Maternal age (y) at } \\
\text { time of presentation }\end{array}$ & $32.5(5.3)$ & $32.6(5.9)$ & $\begin{array}{l}1.00(0.98- \\
1.02)\end{array}$ \\
\hline $\begin{array}{l}\text { No. of preceding } \\
\text { miscarriages }\end{array}$ & $3.3(1.6)$ & $3.3(1.6)$ & $\begin{array}{l}1.02(0.94- \\
1.10)\end{array}$ \\
\hline $\begin{array}{l}\text { No. of first-trimester } \\
\text { pregnancy losses }\end{array}$ & $2.98(1.7)$ & $3.0(1.6)$ & $\begin{array}{l}1.02(0.94- \\
1.10)\end{array}$ \\
\hline $\begin{array}{l}\text { No. of second- } \\
\text { trimester losses }\end{array}$ & $0.29(0.6)$ & $0.29(0.7)$ & $\begin{array}{l}1.22) \\
1.06(0.92- \\
1.21)\end{array}$ \\
\hline $\begin{array}{l}\text { No. of preceding live } \\
\text { births }\end{array}$ & $0.68(0.9)$ & $0.72(0.9)$ & \\
\hline
\end{tabular}


Boogaard, E. van den, Cohn, D.M., Korevaar, J.C., Dawood, F., Vissenberg, R., Middeldorp, S., Goddijn, M., Farquharson, R.G. Number and sequence of preceding miscarriages and maternal age for the prediction of antiphospholipid syndrome in women with recurrent miscarriage 7

Fertility and Sterility: 2013, 99(1), 188-192

\begin{tabular}{|l|l||l|l|}
\hline Independent variable & $\begin{array}{c}\text { Women with } \\
\text { APS (n= 301) }\end{array}$ & $\begin{array}{c}\text { Women with } \\
\text { unexplained RM } \mathbf{( n}= \\
\mathbf{1 , 4 0 7})\end{array}$ & $\begin{array}{l}\text { Odds ratio } \\
\mathbf{( 9 5 \% ~ C I ) ~}\end{array}$ \\
\hline $\begin{array}{l}\geq 1 \text { preceding live birth } \\
(>24 \text { wk), n (\%) }\end{array}$ & $167(53.5)$ & $698(49.6)$ & $\begin{array}{l}1.17(0.91- \\
1.50)\end{array}$ \\
\hline
\end{tabular}

Note: Values are mean (SD) unless ontherwise indicated.

a

Univariable logistic regression analysis.

\section{[TABLE 2]}

Sequence of preceding miscarriages in women with antiphospholipid syndrome (APS) and unexplained recurrent miscarriage (RM).

\begin{tabular}{|c|c|c|c|}
\hline Sequence of pregnancies & $\begin{array}{c}\text { Women with } \\
\text { APS, n (\%) }\end{array}$ & $\begin{array}{c}\text { Women with } \\
\text { unexplained RM, n } \\
(\%)\end{array}$ & $\begin{array}{l}\text { Odds ratio } \\
(95 \% \\
C I)^{a}\end{array}$ \\
\hline $\begin{array}{l}\text { Women with history of } \geq 2 \\
\text { consecutive miscarriages ( } \mathrm{n} \\
=1,526 \text { ) }\end{array}$ & $257 / 265$ (97) & $\mid 1,233 / 1,261(98)$ & $\begin{array}{l}0.73(0.33- \\
1.62)\end{array}$ \\
\hline $\begin{array}{l}\text { Women with history of } \geq 3 \\
\text { consecutive miscarriages (n } \\
=918 \text { ) }\end{array}$ & $140 / 159$ (88) & ||681/759 (90) & $\begin{array}{l}0.84(0.50- \\
1.44)\end{array}$ \\
\hline
\end{tabular}

Univariable logistic regression analysis. 
Boogaard, E. van den, Cohn, D.M., Korevaar, J.C., Dawood, F., Vissenberg, R., Middeldorp, S." Goddijn, M., Farquharson, R.G. Number and sequence of preceding miscarriages and maternal age for the prediction of antiphospholipid syndrome in women with recurrent miscarriage

Fertility and Sterility: 2013, 99(1), 188-192

\section{FIGURE 1}

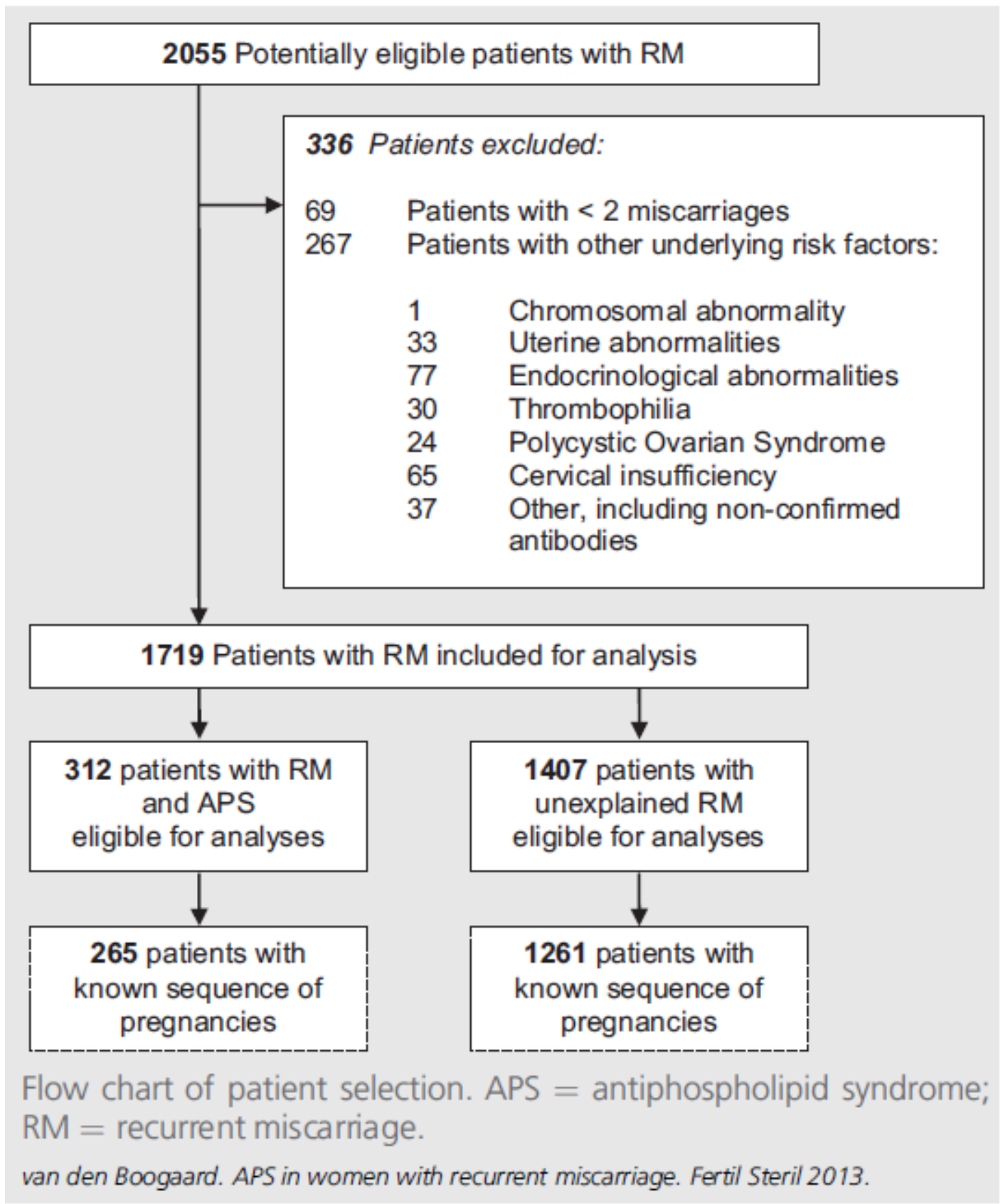

